\title{
Effect of Oral Semaglutide on the Pharmacokinetics of Lisinopril, Warfarin, Digoxin, and Metformin in Healthy Subjects
}

\author{
Tine A. Bækdal ${ }^{1}$. Jeanett Borregaard ${ }^{1}$. Cilie W. Hansen ${ }^{1} \cdot$ Mette Thomsen ${ }^{1}$ - Thomas W. Anderson ${ }^{1}$
}

Published online: 4 April 2019

(c) The Author(s) 2019

\begin{abstract}
Background Oral semaglutide is a tablet co-formulation of the human glucagon-like peptide-1 (GLP-1) analog semaglutide with the absorption enhancer sodium $N$-(8-[2-hydroxybenzoyl] amino) caprylate (SNAC). The absorption of coadministered oral drugs may be altered due to enhancement by SNAC, potential gastric emptying delay by semaglutide, or other mechanisms. Two one-sequence crossover trials investigated the effect of oral semaglutide on the pharmacokinetics of lisinopril, warfarin, digoxin, and metformin.

Methods In trial 1, 52 healthy subjects received lisinopril (20 mg single dose) or warfarin ( $25 \mathrm{mg}$ single dose) with subsequent coadministration with SNAC alone (300 mg single dose), followed by oral semaglutide $20 \mathrm{mg}$ once daily (steady state). In trial 2, 32 healthy subjects received digoxin (500 $\mu \mathrm{g}$ single dose) or metformin ( $850 \mathrm{mg}$ twice daily for 4 days), with subsequent coadministration with SNAC alone followed by oral semaglutide, as in trial 1.

Results There were no apparent effects of oral semaglutide on area under the plasma concentration-time curve (AUC) and maximum plasma concentration $\left(C_{\max }\right)$ for lisinopril, warfarin, and digoxin. The AUC of metformin was increased by $32 \%$ (90\% confidence interval 1.23-1.43) by oral semaglutide coadministration versus metformin alone, whereas the $C_{\max }$ was unaffected. SNAC alone did not affect exposure of lisinopril, warfarin, digoxin, or metformin. Adverse events were in line with those previously observed for GLP-1 receptor agonists.

Conclusions Oral semaglutide or SNAC alone did not appear to affect the exposure of lisinopril, warfarin, or digoxin, and, based on its wide therapeutic index, the higher metformin exposure with oral semaglutide was not considered clinically relevant.
\end{abstract}

\section{Introduction}

Glucagon-like peptide-1 receptor agonists (GLP-1RAs) are effective therapies for the treatment of type 2 diabetes (T2D) and are all currently available as injectable formulations. An oral formulation may improve acceptance and adherence [1] for some patients compared with injectable formulations. The GLP-1 analog semaglutide has been shown to significantly improve glycemic control and reduce body weight as a once-weekly subcutaneous injection in patients with T2D [2-4], and a novel once-daily oral formulation of semaglutide is in development. Oral semaglutide is a co-formulation of

Electronic supplementary material The online version of this article (https://doi.org/10.1007/s40262-019-00756-2) contains supplementary material, which is available to authorized users.

Tine A. Bækdal

tabq@novonordisk.com

1 Novo Nordisk A/S, Vandtårnsvej 108-110, 2860 Søborg, Denmark

\section{Key Points}

Oral semaglutide had no clinically relevant effect on the exposure of lisinopril, warfarin, digoxin, and metformin, which are all commonly used in patients with type 2 diabetes.

Oral semaglutide was found to be well tolerated in combination with these drugs and no new safety issues were identified.

These findings suggest that lisinopril, warfarin, digoxin, and metformin require no dose adjustment when coadministered with oral semaglutide.

semaglutide with an absorption enhancer, sodium $\mathrm{N}-(8-[2-$ hydroxybenzoyl] amino) caprylate (SNAC), which promotes absorption of semaglutide across the gastric mucosa via effects on transcellular pathways [5-7]. Oral semaglutide may alter the absorption of concomitantly administered oral drugs, potentially due to SNAC-mediated absorption enhancement 
in the gastrointestinal tract, delay of gastric emptying by semaglutide, or by other mechanisms. It is therefore important to understand potential drug-drug interactions (DDIs) with oral semaglutide.

Two trials were conducted to investigate the effects of oral semaglutide on the pharmacokinetics (PK) of four cardiovascular and glucose-lowering medications with different solubilities and permeabilities based on the Biopharmaceutics Classification System (BCS) [8]. Lisinopril is an antihypertensive agent that may be used in patients with T2D. It is a BCS class III compound (high solubility and low permeability) [9] with incomplete absorption (approximately 25\%) [9, 10], making it a suitable drug to investigate the potential impact of SNAC on the absorption of concomitant oral medications. Warfarin is a BCS class I/II compound (unconfirmed solubility and high permeability) [11], which is completely absorbed [12], while digoxin is a BCS class IV compound (low solubility and low permeability) with incomplete absorption $(\sim 70 \%)[9,13]$. Both warfarin and digoxin are cardiovascular treatments that may be used in patients with diabetes, and both have narrow therapeutic windows. Metformin (BSC class III, high solubility and low permeability [11]) is a commonly used glucose-lowering agent for the treatment of T2D and is absorbed in the small intestine [14], with a bioavailability of 50-60\% [15]. Absorption of metformin may be reduced and delayed by food [16] and may differ following coadministration with compounds that affect gastric emptying [14]. For drugs with incomplete absorption, coadministration with an absorption enhancer, such as SNAC, may theoretically increase absorption.

\section{Methods}

\subsection{Trial Design and Populations}

Two open-label, one-sequence crossover trials investigated the effect of once-daily oral semaglutide $20 \mathrm{mg}$, at steady state, on the PK of single doses of lisinopril, warfarin, and digoxin, and on the PK of metformin at steady state. In both trials, male and female subjects aged 18-75 years with a body mass index of $20.0-29.9 \mathrm{~kg} / \mathrm{m}^{2}$ were eligible to participate if they were in good general health. The use of healthy subjects is in line with regulatory recommendations for DDI studies $[17,18]$ and is consistent with other DDI trials $[12,19]$. Key exclusion criteria were any clinically significant concomitant disease or disorder, clinically significant abnormal values in clinical laboratory screening tests, any history of gastrointestinal surgery (except uncomplicated surgical procedures), or current smokers smoking more than five cigarettes (or equivalent) per day. All subjects provided written, informed consent. The relevant independent Ethics Committee (Landesamt für Gesundheit und Soziales EthikKommision des Landes Berlin) reviewed and approved the protocol and the trial was conducted in accordance with Good Clinical Practice, the Declaration of Helsinki, and US FDA and European Medicines Agency guidelines for DDI studies $[17,18]$.

\subsection{Treatments}

Doses of victim drugs were selected to represent those likely to maximize the possibility of identifying a DDI, with considerations regarding safety, in line with the FDA DDI guidelines [17]. The doses of semaglutide used were those shown to be effective in a multiple-dose study [20] and a phase II trial [7]. The dose of SNAC was the same as that present in oral semaglutide and the dose shown to provide optimal absorption [5].

\subsubsection{Trial 1 (Lisinopril/Warfarin; NCT02070510)}

Subjects received a single dose of lisinopril $20 \mathrm{mg}$ (Lisinopril-Actavis ${ }^{\circledR}$ ) on day 1 and a single dose of warfarin $25 \mathrm{mg}$ (Coumadin ${ }^{\circledR}$ ) on day 8 (Fig. 1a). The dose of warfarin was the same as that used in other DDI studies with GLP-1 receptor agonists $[12,19]$. The length of the washout periods was based on the half-lives of lisinopril $(12 \mathrm{~h})$ and warfarin (approximately 20-60 h). A single dose of SNAC (300 mg in a tablet) was administered concomitantly with lisinopril $20 \mathrm{mg}$ on day 15 and warfarin $25 \mathrm{mg}$ on day 22 . On day 29 , subjects received once-daily oral semaglutide $5 \mathrm{mg}$ for 1 week, followed by $10 \mathrm{mg}$ for 1 week and $20 \mathrm{mg}$ for 4 weeks to reach steady state (day 70), with a final dose on day 84. Dose escalation of oral semaglutide was implemented to reduce the risk of gastrointestinal adverse events. Once oral semaglutide reached steady state, subjects received a single dose of lisinopril $20 \mathrm{mg}$ (on day 71), followed by a single dose of warfarin $25 \mathrm{mg}$ (on day 78), both concomitantly with oral semaglutide. After each lisinopril and warfarin dose, PK samples were collected over 60 and 168 h, respectively (Electronic Supplementary Table 1). After the last dose of oral semaglutide, PK samples were collected for 504 and $24 \mathrm{~h}$ for semaglutide and SNAC assessments, respectively. All doses were taken in the morning with $120 \mathrm{~mL}$ of water and after an overnight fast ( $\geq 6 \mathrm{~h}$ and no fluid intake occurring within the $2 \mathrm{~h}$ before dosing), followed by post-dose fasting for an additional $30 \mathrm{~min}$.

\subsubsection{Trial 2 (Digoxin/Metformin; NCT02249910)}

Metformin (Metformin Sandoz ${ }^{\circledR}$ ) was investigated at steady state after seven doses as this was expected to give better gastrointestinal tolerability and reduce the within-subject variability of metformin exposure compared with a single 
(a)

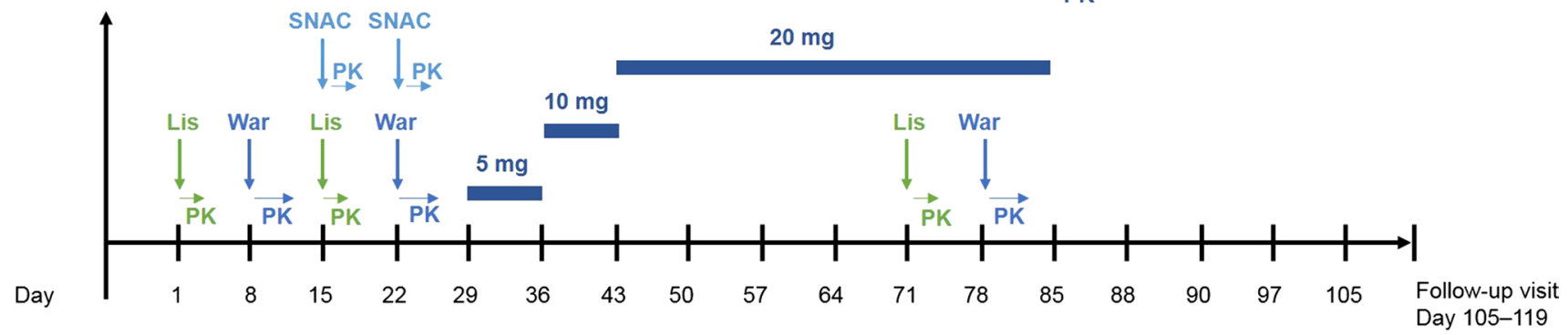

(b)

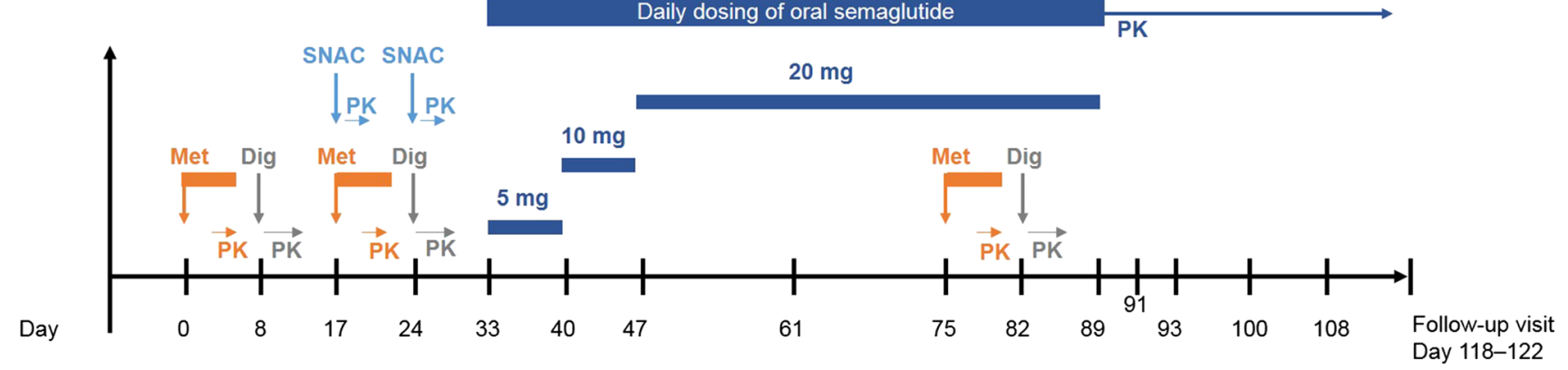

Fig. 1 Design, dosing, and sampling for a trial 1 and b trial 2. a Lisinopril $20 \mathrm{mg}$ and warfarin $25 \mathrm{mg}$ (both analyzed after a single dose). b Digoxin $500 \mu \mathrm{g}$ (single-dose analysis) and metformin

high dose [21]. On days 1-4, subjects were administered metformin $850 \mathrm{mg}$ twice daily for 3 days and once daily on the fourth day, where PK sampling for metformin was performed. On day 8, a single dose of digoxin $\left(\right.$ Digacin $\left.^{\circledR}\right)$ $500 \mu \mathrm{g}$ was administered (Fig. 1b). This single dose was expected to result in an exposure level within the therapeutic range, with a minimum risk of toxicity, and a similar dose has been tested in a similar DDI trial [19]. The length of the washout periods was based on the half-lives of metformin (5-7 h) and digoxin (approximately 30-50 h). From day 17, a similar seven-dose regimen of metformin with single-dose digoxin was repeated. Doses of SNAC (300 mg in tablet form) were administered concomitantly with the four morning metformin doses. On day 24 , subjects received a single dose of SNAC $300 \mathrm{mg}$ concomitantly with a single dose of digoxin $500 \mu \mathrm{g}$. From day 33, subjects received oncedaily oral semaglutide $5 \mathrm{mg}$ for 1 week, followed by $10 \mathrm{mg}$ for 1 week and $20 \mathrm{mg}$ for 4 weeks to reach steady state (to day 74). From day 75, subjects received the final seven doses of metformin, and, on day 82 , subjects received a single dose of digoxin, both concomitantly with oral semaglutide. The last dose of oral semaglutide was on day 87. In each of the three dosing periods, after the last metformin dose and the last digoxin dose, PK samples were collected for 30 and $120 \mathrm{~h}$, respectively (Electronic Supplementary Table 1). After the last dose of oral semaglutide, PK samples were
$850 \mathrm{mg}$ twice daily (steady-state analysis). $P K$ denotes pharmacokinetic sampling, Dig digoxin, Lis lisinopril, Met metformin, SNAC sodium $N$-(8-[2-hydroxybenzoyl] amino) caprylate, War warfarin

collected for up to $840 \mathrm{~h}$ and $24 \mathrm{~h}$ for semaglutide and SNAC assessments, respectively.

All morning doses of the study treatments were taken with $120 \mathrm{~mL}$ of water after an overnight fast $(\geq 6 \mathrm{~h}$ and with no fluid intake within the $2 \mathrm{~h}$ before dosing), followed by post-dose fasting for $30 \mathrm{~min}$. Metformin is usually administered with food but in this trial, the morning dose was administered in the fasting state due to the dosing conditions needed for oral semaglutide. The evening metformin doses were taken $1 \mathrm{~h}$ after dinner.

\subsection{Endpoints and Assessments}

For determination of plasma concentrations, blood samples were drawn at $15 \mathrm{~min}$ before dosing and at frequent time points after dosing based on the drugs' elimination halflives (Electronic Supplementary Table 1). In both trials, total plasma concentrations of semaglutide and SNAC were measured by validated liquid chromatography with tandem mass spectrometry (LC-MS/MS) assays as described previously [22]. Validated LC-MS/MS assays were also used to measure plasma concentrations of lisinopril, S-warfarin and R-warfarin, metformin, and digoxin. In trial 1, the international normalized ratio (INR) was determined from prothrombin time using a validated assay method. 


\subsubsection{Trial 1 (Lisinopril/Warfarin)}

The primary endpoints were area under the plasma concentration-time curve (AUC) from time zero to infinity $\left(\mathrm{AUC}_{0 \text {-inf }}\right)$ for lisinopril, S-warfarin, and R-warfarin after a single dose. Secondary endpoints for lisinopril included AUC from time zero to $60 \mathrm{~h}\left(\mathrm{AUC}_{0-60 \mathrm{~h}}\right)$, maximum plasma concentration $\left(C_{\max }\right)$, time to maximum plasma concentration $\left(t_{\max }\right)$, and terminal half-life $\left(t_{1 / 2}\right)$ after a single dose, and these were derived after dosing on days 1,15 , and 71 . Secondary endpoints for S-warfarin and R-warfarin included AUC from time zero to $168 \mathrm{~h}\left(\mathrm{AUC}_{0-168 \mathrm{~h}}\right), C_{\max }, t_{\max }$, and $t_{1 / 2}$ after a single dose, and these were derived after dosing on days 8 , 22 , and 78. Secondary pharmacodynamic (PD) endpoints for warfarin were the maximum observed INR from time zero to $144 \mathrm{~h}$ after dosing $\left(\mathrm{INR}_{\max }\right)$, the incremental area under the INR response-time curve from time zero to $144 \mathrm{~h}$ $\left(\mathrm{iAUC}_{0-144 \mathrm{~h}, \mathrm{INR}}\right)$, and the time to maximum INR $\left(\mathrm{t}_{\mathrm{INR}, \max }\right)$.

\subsubsection{Trial 2 (Digoxin/Metformin)}

The primary endpoints were (1) $\mathrm{AUC}_{0 \text {-inf }}$ for digoxin after a single dose, administered alone, with SNAC alone and with oral semaglutide, and (2) $\mathrm{AUC}_{0-12 \mathrm{~h}}$ for metformin at steady state, administered alone, with SNAC alone and with oral semaglutide. Secondary endpoints for metformin and digoxin included $C_{\max }, t_{\max }$, and $t_{1 / 2}$. Endpoints relating to digoxin were derived from 0 to $120 \mathrm{~h}$ after a single dose on days 8, 24, and 82. Endpoints for metformin were derived from concentration-time curves from the last dosing until $30 \mathrm{~h}$ after the last of seven repeated doses of metformin alone, with SNAC or with oral semaglutide at steady state (days 4, 20, and 78, respectively).

\subsubsection{Both Trials}

Secondary endpoints for semaglutide were derived after the last dosing (days 84 and 87 in trials 1 and 2, respectively) and included $\mathrm{AUC}_{0-24 \mathrm{~h}}, C_{\max }, t_{\max }$, and $t_{1 / 2}$ at steady state. Secondary endpoints for SNAC included $\mathrm{AUC}_{0-24 \mathrm{~h}}, C_{\max }$, and $t_{\max }$ and were derived after single doses and at steady state. Safety and tolerability were assessed, including treatment-emergent adverse events (AEs), hypoglycemic episodes, physical examination, electrocardiogram (ECG) readings, vital signs, and laboratory parameters.

\subsection{Analytical and Statistical Methods}

\subsubsection{Trial 1 (Lisinopril/Warfarin) Sample Size Calculations}

Assuming the ratio between AUC values was 1.00, and the within-subject standard deviation (SD) of $\log \left(\mathrm{AUC}_{0 \text {-inf }}\right)$ was 0.36 , then 46 subjects completing the study would yield a power of at least $80 \%$ to conclude 'no effect' of coadministration of oral semaglutide for all three endpoints. Assuming the dropout rate was around $12 \%$, the plan was to include 52 subjects. The within-subject SD for $\log \left(C_{\max }\right)$ for S-warfarin and R-warfarin was estimated at 0.07 , and the within-subject $\mathrm{SD}$ for $\log \left(C_{\max }\right)$ for lisinopril was estimated at 0.35 . With 46 completing subjects and an assumed ratio between $C_{\max }$ (with/ without oral semaglutide coadministration) of 1.00 , the marginal power for the secondary endpoints of $C_{\max }$ for R-warfarin and $\mathrm{S}$-warfarin was above $99 \%$, while the marginal power for $C_{\max }$ for lisinopril was $83 \%$.

\subsubsection{Trial 2 (Digoxin/Metformin) Sample Size Calculations}

Assuming the ratio between AUC values was 1.03, and the number of subjects where both primary endpoints were evaluable was 28 , then the combined power of concluding 'no effect' of coadministration of oral semaglutide for both endpoints was at least $95 \%$. Assuming the dropout rate was around $12 \%$, the plan was to include 32 subjects. The within-subject $\mathrm{SD}$ for $\log \left(C_{\mathrm{max}}\right)$ for digoxin (single dose) was estimated at 0.25 , and the within-subject SD for $\log \left(C_{\max }\right)$ for metformin (steady state) was estimated at 0.18 . With 28 completing subjects and an assumed ratio between $C_{\max }$ (with/without oral semaglutide coadministration) of 1.03 , the combined power for both primary endpoints as well as the two secondary endpoints ( $C_{\max }$ for both metformin and digoxin) was $81 \%$ (assuming independence of all four endpoints).

\subsubsection{Definition of Analysis Sets and Statistical Analysis of Endpoints}

PK, PD, and safety assessments were based on all subjects who received at least one dose of the study drug. The primary endpoints were determined using standard non-compartmental methods, and using the linear trapezoidal method based on observed concentrations and actual sampling time points for the calculation of AUC values. Analyses were performed (SAS version 9.3; SAS Software Inc., Cary, NC, USA) using analysis of variance models, with the log-transformed endpoint as the dependent variable, and subject and oral semaglutide or SNAC coadministration (with/without) as fixed effects. For each compound, a lack of an effect of oral semaglutide was concluded if the $90 \%$ confidence interval (CI) for the ratio of AUC or $C_{\max }$ (with/without oral semaglutide) was within 0.80-1.25. Similar analyses were performed for each compound, with or without SNAC. In trial 1, the INR endpoints were analyzed using the same model as that used for the primary endpoints. Secondary endpoints, including $t_{\max }$ and $t_{1 / 2}$ of victim drugs, oral semaglutide, and SNAC, were analyzed descriptively.

In trial 1,12 subjects were incorrectly administered warfarin $5 \mathrm{mg}$ (instead of $25 \mathrm{mg}$ ) with SNAC $300 \mathrm{mg}$ on 
day 22 . A sensitivity analysis excluding those 12 subjects, from day 22, was performed and is reported.

\section{Results}

\subsection{Subjects}

Fifty-two subjects received oral semaglutide and 46 completed trial 1 . Three subjects withdrew due to AEs (described below) and three subjects opted to withdraw themselves for other reasons not related to AEs. In trial 2, 32 subjects received oral semaglutide and all completed the trial, except one subject who was withdrawn prematurely due to four non-serious AEs (described below).

Demographics and baseline clinical characteristics are presented in Table 1.

\subsection{Trial 1}

\subsubsection{Lisinopril}

Mean concentration-time profiles after a single dose of lisinopril appeared to be similar, with or without oral semaglutide (Fig. 2a). The estimated ratio of the $\mathrm{AUC}_{0 \text {-inf }}$ of lisinopril (with vs. without oral semaglutide) was 1.07 , with the 90\% CI (0.99-1.15) within the prespecified 'no effect' interval of 0.80 and 1.25 (Fig. 3a). Mean $C_{\max }$ was similar with lisinopril alone and when coadministered with oral semaglutide (Fig. 3a). Other secondary endpoints were also similar when lisinopril was administered alone or with oral semaglutide; the median $t_{\max }$ was $6.0 \mathrm{~h}$ in both groups, and the mean $t_{1 / 2}$ was 20 and $18 \mathrm{~h}$, respectively (Electronic Supplementary Table 2).

Mean steady-state concentration-time profiles appeared similar when lisinopril was administered alone or with SNAC (Fig. 2a), and the estimated ratio was 0.95 (90\% CI
0.89-1.01) for $\mathrm{AUC}_{0-\text { inf }}$ (Fig. 3b). Furthermore, $C_{\max }, t_{\max }$, and $t_{1 / 2}$ were also similar when lisinopril was administered alone or with SNAC (Fig. 3b and Electronic Supplementary Table 2).

\subsubsection{Warfarin}

Mean concentration-time profiles for S-warfarin appeared similar when a single dose was administered with or without oral semaglutide (Fig. 2b), with an estimated ratio for $\mathrm{AUC}_{0 \text {-inf }}$ of 1.08, with the $90 \% \mathrm{CI}(1.04-1.12)$ within the prespecified interval, indicating no effect (Fig. 3a). Similar results were observed with R-warfarin (Fig. 3a). Mean $C_{\max }$ was comparable between $\mathrm{S}$-warfarin administered alone or with oral semaglutide (Fig. 3a). Median $t_{\max }$ was $1.0 \mathrm{~h}$ and mean $t_{1 / 2}$ was $38 \mathrm{~h}$, with or without oral semaglutide (Electronic Supplementary Table 2).

Twelve subjects incorrectly received warfarin $5 \mathrm{mg}$ instead of warfarin $25 \mathrm{mg}$ with their SNAC treatment on day 22 of the trial, with one $5 \mathrm{mg}$ tablet being dispensed instead of five $5 \mathrm{mg}$ tablets. These 12 subjects were excluded from the PK analyses in the SNAC period. Mean concentration-time profiles of S-warfarin appeared similar when administered alone or when coadministered with SNAC, with estimated ratios of 1.04 (90\% CI 1.02-1.07) for $\mathrm{AUC}_{0-\text { inf }}$ and 0.94 (90\% CI 0.88-1.01) for $C_{\text {max }}$ (Fig. 3b). Similar results were observed with R-warfarin (Fig. 3b). Comparable $t_{\max }$ and $t_{1 / 2}$ were observed for S-warfarin with or without SNAC (Fig. $3 \mathrm{~b}$ and Electronic Supplementary Table 2). Furthermore, coadministration of warfarin with oral semaglutide or SNAC did not affect the total exposure of S-warfarin when analyzed separately in intermediate metabolizers ( $44.2 \%$ of subjects) or extensive metabolizers (53.8\% of subjects) of S-warfarin.
Table 1 Demographics and baseline characteristics of study subjects

\begin{tabular}{lll}
\hline & Trial 1 & Trial 2 \\
\hline Number of subjects & 52 & 32 \\
Mean age, years (min-max) & $54(22-73)$ & $59(27-73)$ \\
Sex $[n(\%)]$ & & \\
Female & $18(34.6)$ & $12(37.5)$ \\
Male & $34(65.4)$ & $20(62.5)$ \\
Ethnicity $[n(\%)]$ & & $32(100)$ \\
White & $50(96.2)$ & - \\
Other & $2(3.8)$ & $76.0(58.2-104.7)$ \\
Mean body weight, kg (min-max) & $76.9(51.7-102.0)$ & $25.4(21.1-29.3)$ \\
Mean body mass index, kg/m ${ }^{2}(\min -\max )$ & $25.5(20.5-29.8)$ & - \\
International normalized ratio [geometric mean $(\mathrm{CV})]$ & $0.99(4.8)$ & \\
\hline
\end{tabular}

$C V$ coefficient of variation, $\min$ minimum, $\max$ maximum 
Fig. 2 Geometric mean plasma concentration-time profiles of lisinopril, S-warfarin, digoxin, and metformin with or without coadministration of oral semaglutide or SNAC. a Lisinopril (single dose) \pm oral semaglutide \pm SNAC. b S-warfarin (single dose) \pm oral semaglutide \pm SNAC* * * Excludes the 12 subjects administered a lower dose of warfarin (administration of warfarin $5 \mathrm{mg}$ instead of $25 \mathrm{mg}$ ) during coadministration with SNAC. c Digoxin (single dose) \pm oral semaglutide \pm SNAC. $d$

Metformin (steady state) \pm oral semaglutide \pm SNAC. SNAC sodium $N$-(8-[2-hydroxybenzoyl] amino) caprylate
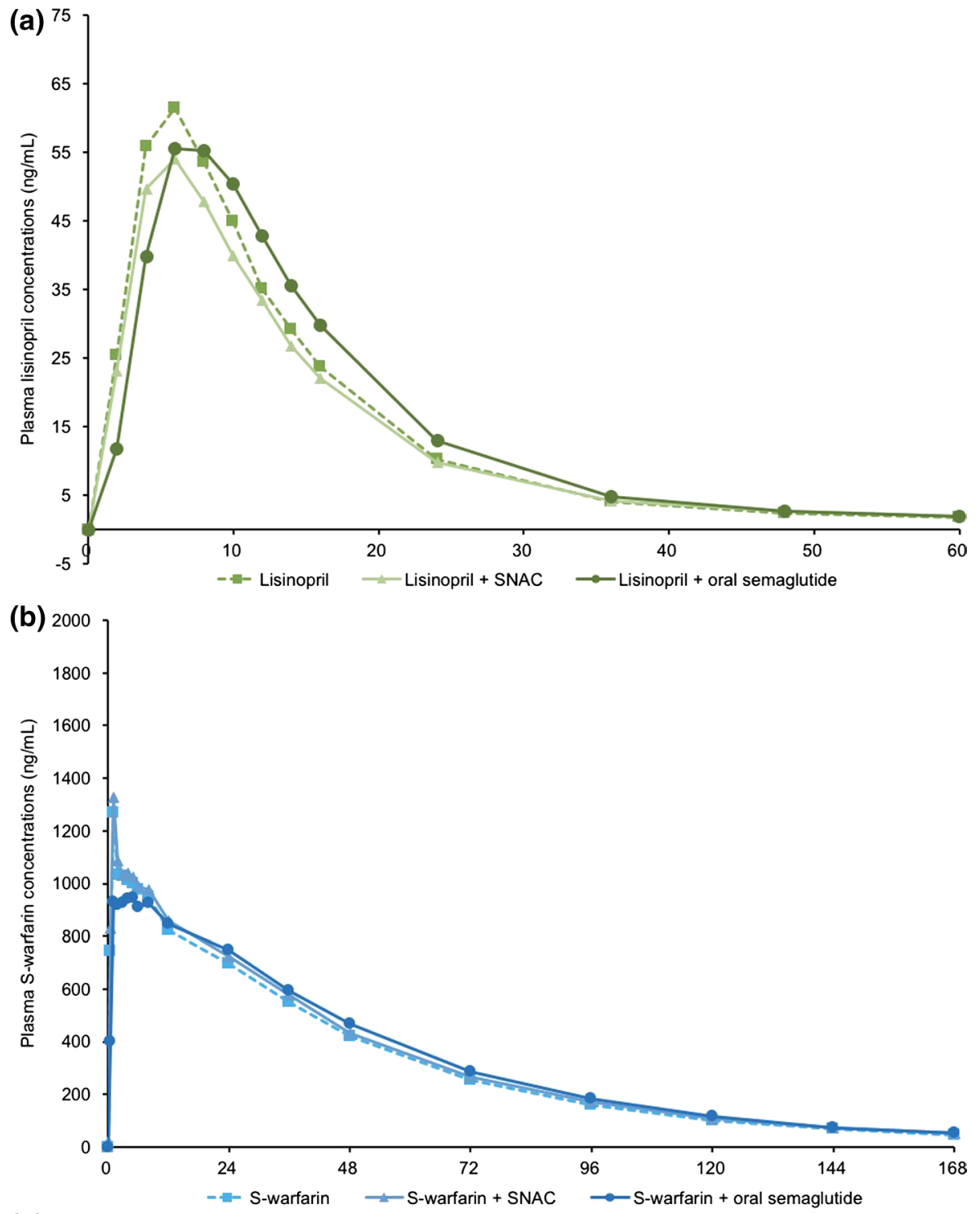

(c)

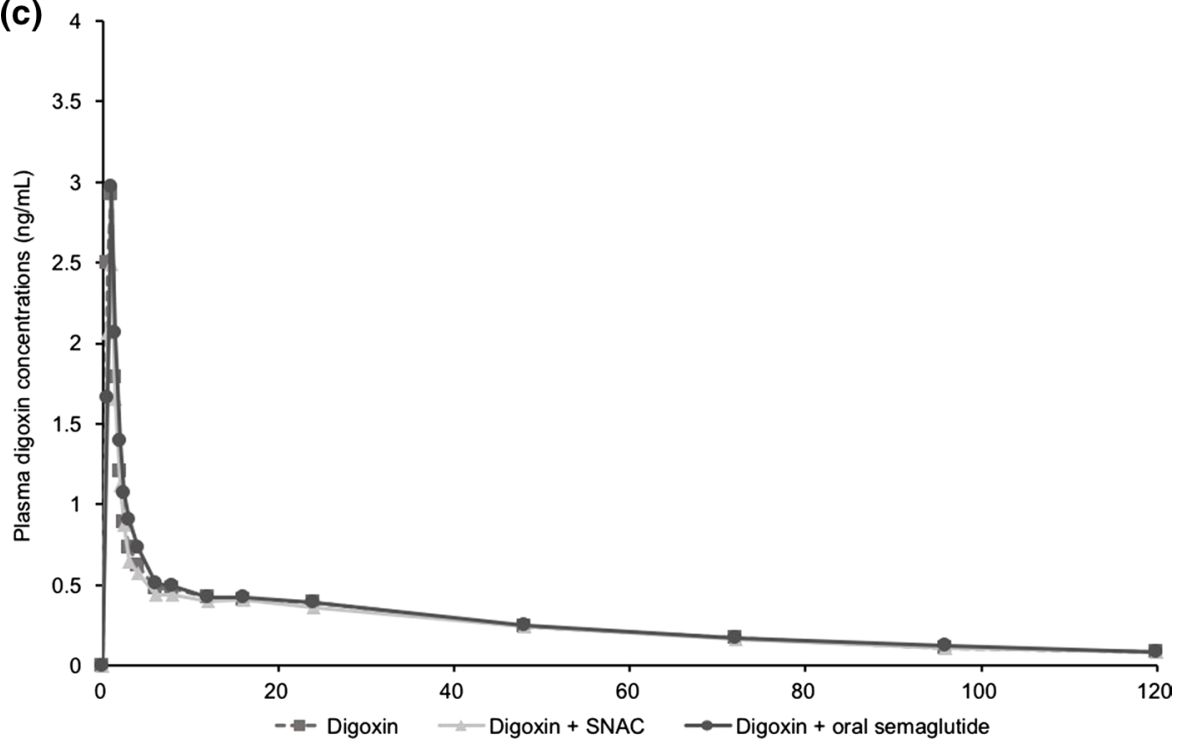


Fig. 2 (continued)

(d)

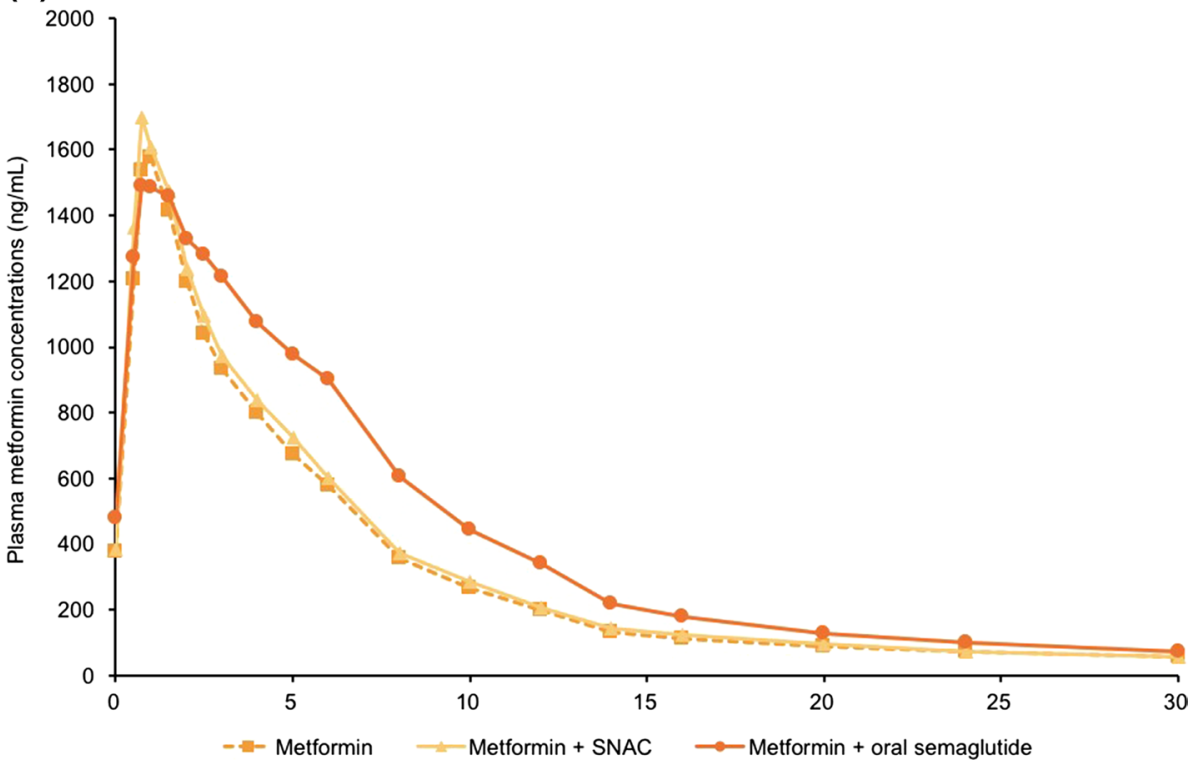

\subsubsection{Semaglutide and Sodium $N$-(8-[2-Hydroxybenzoyl] amino) caprylate (SNAC)}

Mean $\mathrm{AUC}_{0-24 \mathrm{~h}}$ for semaglutide at steady state was 761.96 $\mathrm{nmol} \cdot \mathrm{h} / \mathrm{L}$ and $C_{\max }$ was $37.35 \mathrm{nmol} / \mathrm{L}$. The median $t_{\max }$ of semaglutide was $1.0 \mathrm{~h}$ and mean $t_{1 / 2}$ was $162 \mathrm{~h}$. $\mathrm{AUC}_{0-24 \mathrm{~h}}$, $C_{\max }, t_{\max }$, and $t_{1 / 2}$ for SNAC appeared similar across the five conditions of SNAC administration (SNAC + lisinopril, SNAC + digoxin, oral semaglutide + lisinopril, oral semaglutide + digoxin, or oral semaglutide alone) (Electronic Supplementary Table 3).

\subsubsection{Pharmacodynamics}

Coadministration of warfarin with oral semaglutide at steady state did not affect $\mathrm{INR}_{\max }$; the estimated ratio (with vs. without oral semaglutide) was 0.98 (90\% CI 0.96-1.01). Median time to $\mathrm{INR}_{\max }$ was $35.9-47.7 \mathrm{~h}$ across the three conditions of warfarin administration. The iAUC $\mathrm{INR}_{\text {INR }}$ appeared unaffected by semaglutide coadministration, with a ratio of 1.00 (90\% CI 0.93-1.06). Coadministration of warfarin with SNAC alone did not affect $\mathrm{INR}_{\max }$ (estimated ratio 0.85; 90\% CI 0.83-0.88); CIs were within the prespecified interval, indicating no effect. The $\mathrm{iAUC}_{\mathrm{INR}}$ was reduced by $28 \%$ with SNAC alone plus warfarin, and a similar reduction was observed when patients who mistakenly received the lower dose of warfarin were excluded.

\subsection{Trial 2}

\subsubsection{Digoxin}

Mean concentration-time profiles for digoxin appeared similar when a single dose was administered with or without oral semaglutide (Fig. 2c); the estimated ratio of $\mathrm{AUC}_{0-\text { inf }}$ was 1.03 and the $90 \% \mathrm{CI}(0.96-1.11)$ was within the prespecified 'no effect' interval (Fig. 3a). Mean $C_{\max }$ was similar with digoxin alone and when coadministered with oral semaglutide (Fig. 3a). In addition, median $t_{\max }$ was $1.0 \mathrm{~h}$ under both conditions, and the mean $t_{1 / 2}$ was $42-43 \mathrm{~h}$ (Electronic Supplementary Table 2).

Mean concentration-time profiles of digoxin appeared similar when administered alone or with SNAC alone (Fig. 2c). The estimated ratio for $\mathrm{AUC}_{0-\text { inf }}$ was 0.96 and the $90 \%$ CI (0.91-1.01) indicated no effect (Fig. 3b). Mean $C_{\max }$ was lower when digoxin was coadministered with SNAC versus digoxin alone, with an estimated ratio of 0.82 and the $90 \% \mathrm{CI}(0.73-0.92)$ outside the prespecified interval (Fig. 3b). However, $t_{\max }$ and $t_{1 / 2}$ of digoxin were similar when administered with or without SNAC (Electronic Supplementary Table 2).

\subsubsection{Metformin}

Mean steady-state concentration-time profiles for metformin, with or without oral semaglutide, are presented in Fig. 2d. $A_{U C} C_{0-12 \mathrm{~h}}$ was higher when metformin was coadministered with oral semaglutide compared with when metformin was administered alone; the estimated ratio was 1.32 
Fig. 3 Estimated AUC and $C_{\max }$ ratios (with $90 \% \mathrm{CI}$ ) for lisinopril, warfarin*, digoxin, and metformin with or without coadministration of a oral semaglutide or b SNAC. * Excludes the 12 subjects administered $5 \mathrm{mg}$ warfarin instead of $25 \mathrm{mg}$ during coadministration with SNAC. $A U C_{0-12 h}$ area under the concentration-time curve from time zero to $12 \mathrm{~h}, A U C$ 0 -inf area under the concentration-time curve from time zero to infinity, $C I$ confidence interval, $C_{\max }$ maximum plasma concentration, SNAC sodium $N$-(8-[2-hydroxybenzoyl] amino) caprylate (a) Lisinopril
\pm semaglutide
$n=46$

S-warfarin \pm semaglutide $n=46$

\section{R-warfarin \pm semaglutide $n=46$}

Digoxin \pm semaglutide $n=31$

\section{Metformin \pm semaglutide $n=31$}

Estimate $(90 \% \mathrm{Cl})$

$1.07(0.99-1.15)$

$0.96(0.88-1.06)$

$1.08(1.04-1.12)$

$0.88(0.83-0.94)$

1.11 (1.06-1.15)

$0.91(0.86-0.96)$

$1.03(0.96-1.11)$

$0.98(0.89-1.09)$

1.32 (1.23-1.43)

$0.98(0.90-1.06)$

Estimate $(90 \% \mathrm{Cl})$

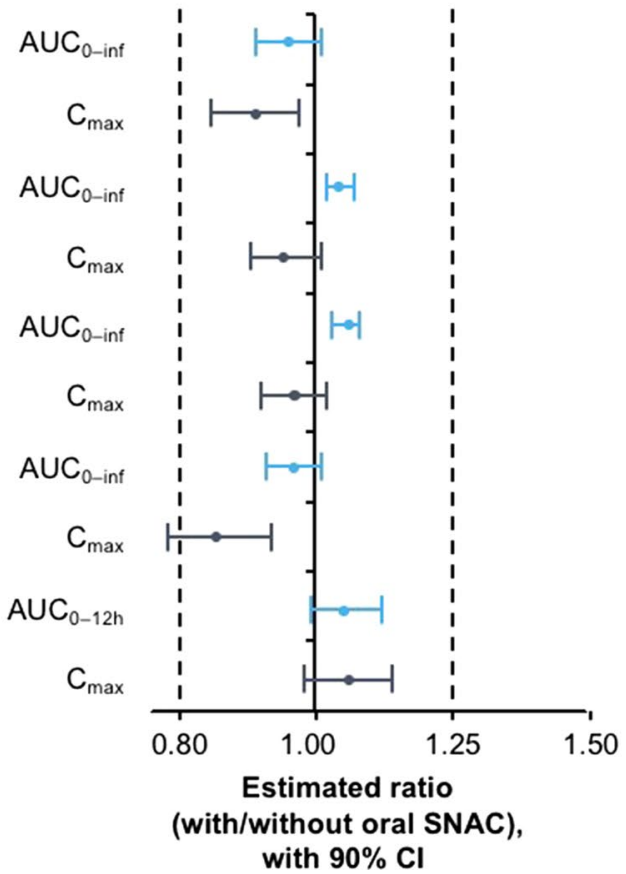

$0.95(0.89-1.01)$

$0.89(0.81-0.97)$

$1.04(1.02-1.07)$

$0.94(0.88-1.01)$

$1.06(1.03-1.08)$

$0.96(0.90-1.02)$

$0.96(0.91-1.01)$

$0.82(0.73-0.92)$

$1.05(0.99-1.12)$

$1.06(0.98-1.14)$ and the $90 \%$ CI (1.23-1.43) was not within the prespecified 'no effect' interval of 0.80 and 1.25 (Fig. 3a). Mean $C_{\max }$ was similar with metformin alone and when coadministered with oral semaglutide (Fig. 3a). In addition, $t_{\max }$ and $t_{1 / 2}$ were similar with metformin alone or when coadministered with oral semaglutide; the median $t_{\max }$ was 0.8 and $1.0 \mathrm{~h}$, respectively, and the mean $t_{1 / 2}$ was 16 and $13 \mathrm{~h}$, respectively (Electronic Supplementary Table 2).

Mean steady-state concentration-time profiles for metformin appeared similar with metformin alone and with SNAC (Fig. 2d), and the estimated ratio was 1.05 (90\% CI 0.99-1.12) (Fig. 3b). In addition, $C_{\max }, t_{\max }$, and $t_{1 / 2}$ were 
similar when metformin was administered with or without SNAC (Fig. 3b and Electronic Supplementary Table 2).

\subsubsection{Semaglutide and SNAC}

For semaglutide at steady state, the range of geometric means was $622-719 \mathrm{nmol} \cdot \mathrm{h} / \mathrm{L}$ for $\mathrm{AUC}_{0-24 \mathrm{~h}}$ and $31.0-35.8 \mathrm{nmol} / \mathrm{L}$ for $C_{\max }$; median $t_{\max }$ was $1.0-1.5 \mathrm{~h}$ and the geometric mean $t_{1 / 2}$ was $162 \mathrm{~h}$.

$\mathrm{AUC}_{0-24 \mathrm{~h}}, C_{\max }, t_{\max }$, and $t_{1 / 2}$ for SNAC appeared similar across the five conditions of SNAC administration (Electronic Supplementary Table 3b).

\subsection{Safety and Tolerability}

During the total semaglutide dosing phase, 228 AEs were reported in $74 \%(36 / 49)$ of subjects in trial 1, and 151 AEs were reported in $84 \%$ (27/32) of subjects in trial 2 (Table 2). The most frequent AEs were gastrointestinal events. The majority of AEs were rated as mild or moderate in severity by the investigator. There were no severe events in Trial 1 and only one event in trial 2 (syncope in one subject, which was evaluated as unlikely to be related to the trial products by the investigator). In trial 1 , one subject experienced a bleeding event (epistaxis) after warfarin $(25 \mathrm{mg}$ ) dosing on day 9 , and this was of moderate severity and deemed possibly related to warfarin $\left(\mathrm{INR}_{\max }\right.$ was 2.53$)$. An overview of AEs by treatment is provided in Electronic Supplementary Table 4.

There were no serious adverse events and no deaths. In trial 1, three subjects withdrew due to AEs (gastroduodenitis on day 3, ankle fracture on day 17 , and herpes zoster infection on day 36 following treatment with lisinopril, lisinopril plus SNAC, and oral semaglutide, respectively). In trial 2, one subject withdrew due to four non-serious AEs (nausea, vomiting, eructation, and dyspepsia) after 6 days of treatment with oral semaglutide $10 \mathrm{mg}$.

No severe hypoglycemic episodes were reported. There were no clinically relevant changes in physical examination, ECG, vital signs, or mean clinical laboratory values, although expected GLP-1RA class effects [decrease in systolic blood pressure and body weight (Electronic Supplementary Figs. 1 and 2), increase in pulse rate, and increases in lipase and amylase] were observed with semaglutide.

\section{Discussion}

Given the absorption-enhancement properties of SNAC and/ or the potential delaying effect of semaglutide on gastric emptying, the current trials were conducted to evaluate the
Table 2 Treatment-emergent adverse events in healthy subjects following oral semaglutide treatment

\begin{tabular}{lll}
\hline & Trial 1 & Trial 2 \\
\hline Number of subjects & 49 & 32 \\
Total events $[n(\%), E]$ & $36(73.5), 228$ & $27(84.4), 151$ \\
Serious events & 0 & 0 \\
Adverse events occurring in $>10 \%$ & & \\
of subjects in either trial $[n(\%), E]$ & & \\
Decreased appetite & $14(28.6), 17$ & $21(65.6), 22$ \\
Nausea & $20(40.8), 35$ & $12(37.5), 21$ \\
Diarrhea & $8(16.3), 16$ & $12(37.5), 18$ \\
Fatigue & $14(28.6), 14$ & $4(12.5), 5$ \\
Headache & $9(18.4), 11$ & $7(21.9), 17$ \\
Dyspepsia & $9(18.4), 14$ & $7(21.9), 8$ \\
Vomiting & $7(14.3), 23$ & $6(18.8), 8$ \\
Eructation & $4(8.2), 9$ & $8(25.0), 8$ \\
Abdominal pain & $7(14.3), 9$ & $5(15.6), 5$ \\
Dizziness & $11(22.4), 14$ & 0 \\
Abdominal distension & $7(14.3), 8$ & $3(9.4), 3$ \\
Abdominal discomfort & $5(10.2), 5$ & $4(12.5), 5$ \\
Malaise & $7(14.3), 9$ & $1(3.1), 1$ \\
Nasopharyngitis & $1(2.0), 1$ & $6(18.8), 6$ \\
Constipation & $5(10.2), 9$ & $1(3.1), 1$ \\
\hline
\end{tabular}

$E$ number of adverse events, $n$ number of subjects with an adverse event, \% proportion of subjects having an adverse event

possibility of DDIs between oral semaglutide and agents of different BCS classes that are used in the management of $\mathrm{T} 2 \mathrm{D}$ and associated comorbidities.

No apparent changes in AUC or $C_{\max }$ were observed for single doses of lisinopril and warfarin in the presence of oral semaglutide or SNAC alone. Moreover, oral semaglutide at steady state had no influence on the INR response to warfarin when administered simultaneously. Similarly, in a PK study by Hausner et al. [19], there was no effect of subcutaneous semaglutide on warfarin exposure or INR.

Delayed gastric emptying could give medications with low solubility (such as digoxin) additional time to dissolve and be absorbed, which may increase exposure. Previous PK studies have observed a trend to slight reductions in $C_{\max }$ and delayed $t_{\max }$ with digoxin in the presence of some GLP-1RAs [9, 23]. For example, in a study of the effect of exenatide on digoxin PK, AUC was unchanged but $C_{\max }$ decreased by $17 \%$ [23]. Furthermore, coadministration with liraglutide has been reported to reduce the $C_{\max }$ of digoxin by $27-38 \%$ [9]. However, in the current study, oral semaglutide or SNAC alone had no notable effects on the exposure to digoxin following a single dose. Consistent with these findings, a PK study by Hausner et al. [19] has noted that the AUC and $C_{\max }$ of single-dose digoxin were unaffected by coadministration of subcutaneous semaglutide. 
Oral semaglutide at steady state increased the total steady-state exposure of metformin by $32 \%$. However, based on the wide therapeutic index of metformin, the slightly greater exposure when coadministered with oral semaglutide was not considered to be clinically relevant. Importantly, $C_{\max }, t_{\max }$, and $t_{1 / 2}$ for metformin were not affected by coadministration with oral semaglutide. Reassuringly, SNAC had no effect on exposure to metformin and therefore the slight increase in metformin exposure may be due to the GLP-1RA component rather than SNAC. In the study by Hausner et al. [19], where metformin was coadministered with subcutaneous semaglutide, there appeared to be no effect on AUC or $C_{\max }$; however, an increase in AUC of up to $15 \%$ has been reported following coadministration of multiple doses of dulaglutide with steady-state metformin [24]. The exact mechanism responsible for the higher AUC of metformin with oral semaglutide and dulaglutide is unknown but may relate to a delay in gastric emptying or differences in study design. Metformin is primarily absorbed in the small intestine and delayed gastric emptying with GLP-1RAs might result in slower and more prolonged absorption [25]. Metformin is usually administered with food, primarily to aid tolerability. In the present study, metformin was administered with food in the evening, but was administered in a fasting state in the morning in order to preserve the dosing conditions for oral semaglutide. Since the dosing conditions remained the same throughout the study, whether or not SNAC or oral semaglutide was administered, this difference between evening and morning metformin dosing is unlikely to have affected the study outcomes. The $t_{\max }$ for metformin was shorter $(0.8-1.0 \mathrm{~h})$ in trial 2 than is generally reported $(2.5 \mathrm{~h})$ [26]. The reason for this is unknown; however, food taken around the time of metformin dosing results in delayed $t_{\max }$ and reduced AUC [16]. Therefore, the shorter $t_{\max }$ may reflect the fasting periods around oral semaglutide and metformin morning dosing in the current trial. Effects on gastric emptying did not appear to affect the absorption of any of the other drugs studied.

Our data indicate that SNAC does not increase exposure of coadministered drugs, and supports the suggestion that the effect of SNAC requires compounds to be co-formulated, and in close proximity to enhance absorption [5].

The PK results observed in this study for steady-state semaglutide are in line with those observed in a multipledose trial with oral semaglutide [20]. In addition, the disposition is similar to studies with subcutaneous semaglutide [19]. For example, mean $t_{1 / 2}$ was $162 \mathrm{~h}$ in the current trials, $153-161 \mathrm{~h}$ in a multiple-dose trial with oral semaglutide, and $156-160 \mathrm{~h}$ for subcutaneous semaglutide $[19,20]$. Similarly, the safety profile of oral semaglutide in the current trials is consistent with other trials assessing oral [7,27] and subcutaneous semaglutide $[2-4,28]$. The most frequently reported AEs with semaglutide were gastrointestinal disorders, which are considered a class effect of GLP-1RAs. No serious AEs, deaths, or severe hypoglycemic episodes were reported. Further safety data on oral semaglutide are being provided [27] by the ongoing PIONEER phase IIIa program being conducted in a broad population of subjects with T2D.

Potential limitations of the current trials include the incorrect dosing of warfarin in 12 subjects in Trial 1. The exclusion of these subjects and the resultant reduction in sample size may have impacted PK/PD conclusions related to warfarin coadministration with SNAC alone. Another potential limitation is that the trials had a one-sequence crossover design, and differences in observation/exposure periods make it difficult to compare safety and tolerability profiles between treatment arms. In addition, the trial used a population of healthy volunteers that was predominantly White and male, and therefore may not be fully representative of a general diabetes population.

\section{Conclusion}

When coadministered, oral semaglutide had no clinically relevant effect on the exposure of lisinopril, warfarin, digoxin, and metformin. Similarly, SNAC alone did not increase the absorption of the victim drugs. Findings suggest that these drugs, which are all commonly used in patients with T2D, require no dose adjustment when coadministered with oral semaglutide. Oral semaglutide was found to be well tolerated in combination with lisinopril, warfarin, digoxin, and metformin, and no new safety issues were identified.

Acknowledgements These trials were sponsored by Novo Nordisk A/S. Writing support was provided by Graham Allcock and Emma Marshman of Spirit Medical Communications Ltd, supported by Novo Nordisk A/S in accordance with Good Publication Practice (GPP3) guidelines (http://www.ismpp.org/gpp3). The authors would like to thank all the participants, investigators and trial-site staff who were involved in the conduct of the trials. The authors would also like to thank Salvatore Calanna (Novo Nordisk A/S) for review and input into the manuscript.

\section{Compliance with Ethical Standards}

Conflict of interest Tine A. Bækdal, Mette Thomsen, and Thomas W. Anderson are employees of, and own stock, in Novo Nordisk A/S. Jeanett Borregaard is an employee of Novo Nordisk A/S. Cilie W. Hansen was an employee of Novo Nordisk A/S at the time of writing this manuscript.

Funding These trials were sponsored by Novo Nordisk A/S, Søborg, Denmark.

Author contributions All authors participated in the trial design, were involved in data analysis and interpretation, and participated in writing the manuscript, together with medical writing services provided by the sponsor. All authors have approved the submitted manuscript. 
Ethical approval All procedures performed in studies involving human participants were in accordance with the ethical standards of the institutional and/or national research committee and with the 1964 Helsinki declaration and its later amendments or comparable ethical standards.

Consent to participate Informed consent was obtained from all individual participants included in these trials.

Data availability The datasets generated and/or analyzed during the current study are available from the corresponding author upon reasonable request.

Open Access This article is distributed under the terms of the Creative Commons Attribution-NonCommercial 4.0 International License (http://creativecommons.org/licenses/by-nc/4.0/), which permits any noncommercial use, distribution, and reproduction in any medium, provided you give appropriate credit to the original author(s) and the source, provide a link to the Creative Commons license, and indicate if changes were made.

\section{References}

1. Cooke CE, Lee HY, Tong YP, et al. Persistence with injectable antidiabetic agents in members with type 2 diabetes in a commercial managed care organization. Curr Med Res Opin. 2010;26:231-8.

2. Ahrén B, Masmiquel L, Kumar H, et al. Efficacy and safety of once-weekly semaglutide versus once-daily sitagliptin as an add-on to metformin, thiazolidinediones, or both, in patients with type 2 diabetes (SUSTAIN 2): a 56-week, double-blind, phase $3 \mathrm{a}$, randomised trial. Lancet Diabetes Endocrinol. 2017;5:341-54.

3. Aroda VR, Bain SC, Cariou B, et al. Efficacy and safety of once-weekly semaglutide versus once-daily insulin glargine as add-on to metformin (with or without sulfonylureas) in insulinnaive patients with type 2 diabetes (SUSTAIN 4): a randomised, open-label, parallel-group, multicentre, multinational, phase $3 \mathrm{a}$ trial. Lancet Diabetes Endocrinol. 2017;5:355-66.

4. Sorli C, Harashima SI, Tsoukas GM, et al. Efficacy and safety of once-weekly semaglutide monotherapy versus placebo in patients with type 2 diabetes (SUSTAIN 1): a double-blind, randomised, placebo-controlled, parallel-group, multinational, multicentre phase 3a trial. Lancet Diabetes Endocrinol. 2017;5:251-60.

5. Buckley ST, Bækdal TA, Vegge A, et al. Transcellular stomach absorption of a derivatized glucagon-like peptide-1 receptor agonist. Sci Transl Med. 2018;10(467):eaar7047.

6. Connor A, Borregaard J, Buckley ST, et al. Site of absorption of an oral formulation of semaglutide. Diabetes. 2017;66(Suppl. 1):1180-P.

7. Davies M, Pieber TR, Artoft-Nielsen ML, et al. Effect of oral semaglutide compared with placebo and subcutaneous semaglutide on glycemic control in patients with type 2 diabetes: a randomized clinical trial. JAMA. 2017;318:1460-70.

8. Amidon GL, Lennernäs H, Shah VP, et al. A theoretical basis for a biopharmaceutic drug classification: the correlation of in vitro drug product dissolution and in vivo bioavailability. Pharm Res. 1995;12:413-20.

9. Malm-Erjefalt M, Ekblom M, Vouis J, et al. Effect on the gastrointestinal absorption of drugs from different classes in the biopharmaceutics classification system, when treating with liraglutide. Mol Pharm. 2015;12:4166-73.
10. Lisinopril $20 \mathrm{mg}$ tablets. Summary of product characteristics. https://www.medicines.org.uk/emc/medicine/24003. Accessed 30 Oct 2018.

11. Lindenberg M, Kopp S, Dressman JB. Classification of orally administered drugs on the World Health Organization Model list of Essential Medicines according to the biopharmaceutics classification system. Eur J Pharm Biopharm. 2004;58:265-78.

12. Soon D, Kothare PA, Linnebjerg H, et al. Effect of exenatide on the pharmacokinetics and pharmacodynamics of warfarin in healthy Asian men. J Clin Pharmacol. 2006;46:1179-87.

13. Digoxin. Summary of product characteristics. https://www. medicines.org.uk/emc/medicine/23944. Accessed 30 Oct 2018.

14. Marathe PH, Wen Y, Norton J, et al. Effect of altered gastric emptying and gastrointestinal motility on metformin absorption. Br J Clin Pharmacol. 2000;50:325-32.

15. Pentikäinen PJ, Neuvonen PJ, Penttilä A. Pharmacokinetics of metformin after intravenous and oral administration to man. Eur J Clin Pharmacol. 1979;16:195-202.

16. Sambol NC, Brookes LG, Chiang J, et al. Food intake and dosage level, but not tablet vs solution dosage form, affect the absorption of metformin $\mathrm{HCl}$ in man. Br J Clin Pharmacol. 1996;42:510-2.

17. Food and Drug Administration. Drug interaction studies—study design, data analysis, implications for dosing and labeling recommendations. https://www.fda.gov/downloads/drugs/guida nces/ucm292362.pdf. Accessed 1 Nov 2018.

18. European Medicines Agency. Guideline on the investigation of drug interactions. http://www.ema.europa.eu/docs/en_GB/ document_library/Scientific_guideline/2012/07/WC50012960 6.pdf. Accessed 30 Sept 2018.

19. Hausner H, Derving Karsbøl J, Holst AG, et al. Effect of semaglutide on the pharmacokinetics of metformin, warfarin, atorvastatin and digoxin in healthy subjects. Clin Pharmacokinet. 2017;56:1391-401.

20. Granhall C, Donsmark M, Blicher TM, et al. Safety and pharmacokinetics of single and multiple ascending doses of the novel oral human GLP-1 analogue, oral semaglutide, in healthy subjects and subjects with type 2 diabetes. Clin Pharmacokinet. 2018. https://doi.org/10.1007/s40262-018-0728-4 (Epub 18 Dec 2018).

21. Graham GG, Punt J, Arora M, et al. Clinical pharmacokinetics of metformin. Clin Pharmacokinet. 2011;50:81-98.

22. Granhall C, Sondergaard FL, Thomsen M, et al. Pharmacokinetics, safety and tolerability of oral semaglutide in subjects with renal impairment. Clin Pharmacokinet. 2018;57:1571-80.

23. Kothare PA, Soon DK, Linnebjerg H, et al. Effect of exenatide on the steady-state pharmacokinetics of digoxin. J Clin Pharmacol. 2005;45:1032-7.

24. TRULICITY $1.5 \mathrm{mg} \& 0.75 \mathrm{mg}$ solution for injection. Summary of product characteristics. https://www.medicines.org.uk/emc/ medicine/29747. Accessed 30 Aug 2018.

25. McCreight LJ, Bailey CJ, Pearson ER. Metformin and the gastrointestinal tract. Diabetologia. 2016;59:426-35.

26. Metformin. Summary of product characteristics. http://www. medicines.org.uk/emc/medicine/23244. Accessed 1 Nov 2018.

27. Haluzik M, Rosenstock J, Terauchi Y, et al. Effect and safety or oral semaglutide monotherapy in type 2 diabetes: PIONEER 1 trial [oral presentation \#38]. Presentation at the European Association for the Study of Diabetes 54th Annual Meeting, 1-5 October 2018, Berlin, Germany.

28. Marso SP, Bain SC, Consoli A, et al. Semaglutide and cardiovascular outcomes in patients with type 2 diabetes. N Engl J Med. 2016;375:1834-44. 